\title{
BILITE: A Bayesian Randomized Phase II Design for Immunotherapy by Jointly Modeling the Longitudinal Immune Response and Time-to-event Efficacy
}

\author{
Beibei Guo ${ }^{1}$, and Yong Zang ${ }^{2,3}$ \\ ${ }^{1}$ Department of Experimental Statistics, Louisiana State University \\ Baton Rouge, LA 70803, U.S.A. \\ email: beibeiguo@lsu.edu \\ ${ }^{2}$ Department of Biostatistics, School of Medicine, Indiana University \\ ${ }^{3}$ Center for Computational Biology and Bioinformatics, Indiana University \\ Indianapolis, IN 46202, U.S.A.
}

\begin{abstract}
Immunotherapy - treatments that target a patient's immune system - has attracted considerable attention in cancer research. Its recent success has led to generation of novel immunotherapeutic agents that need to be evaluated in clinical trials. Two unique features of immunotherapy are the immune response and the fact that some patients may achieve longterm durable response. In this article, we propose a two-arm Bayesian adaptive ran-domized phase II clinical trial design for immunotherapy that jointly models the longitudinal immune response and time-to-event efficacy (BILITE), with a fraction of patients assumed to be cured by the treatment. The longitudinal immune response is modeled using hierarchi-cal nonlinear mixed-effects models with possibly different trajectory patterns for the cured and susceptible groups. Conditional on the immune response trajectory, the time-to-event efficacy data for patients in the susceptible group is modeled via a time-dependent Cox-type regression model. We quantify the desirability of the treatment using a utility function and
\end{abstract}

This is the author's manuscript of the article published in final edited form as:

Guo, B., \& Zang, Y. (2020). BILITE: A Bayesian randomized phase II design for immunotherapy by jointly modeling the longitudinal immune response and time-to-event efficacy. Statistics in Medicine, 39(29), 4439-4451. https://doi.org/10.1002/sim.8733 
propose a two-stage design to adaptively randomize patients to treatments and make treatment recommendations at the end of the trial. Simulation studies show that compared with a conventional design that ignores the immune response, BILITE yields superior operating characteristics in terms of the ability to identify promising agents and terminate the trial early for futility.

KEY WORDS: Immunotherapy; Longitudinal immune response; Cure models; Bayesian adaptive design. 


\section{Introduction}

Cancer immunotherapy is an innovative treatment approach that stimulates a patient's immune system to fight cancer. It has been hailed as the most promising new cancer treatment approach since the development of the first chemotherapies in the late 1940s. ${ }^{1-3}$ The landmark success of immunotherapeutic agents such as ipilimumab and nivolumab has led to intense focus on other co-stimulatory and co-inhibitory immune pathways. This research has produced novel immunotherapies for cancer treatment that need to be evaluated in clinical trials. ${ }^{4}$

Numerous frequentist and Bayesian designs have been proposed for phase II trials. Frequentist designs include the famous Simon's optimal two-stage design ${ }^{5}$ and its extensions. ${ }^{6-11}$ From the Bayesian perspective, decisions are based on the posterior probabilities, ${ }^{12-14}$ predictive probabilities, ${ }^{15,16}$ or Bayes factors. ${ }^{17}$ Recently, randomized phase II trials have become increasingly popular in drug development. ${ }^{4,16,18}$

Unfortunately, these phase II designs were developed mainly for evaluating chemotherapeutic agents so they are inefficient and inappropriate for immunotherapy due to the unique features and mechanisms for immunotherapeutic agents to treat cancer. Unlike chemotherapy that works by shrinking the tumor, immunotherapy targets a patient's immune system so it often delays cancer progression and prolongs survival without significant tumor shrinkage, and some patients who receive immunotherapy may achieve long-term durable response. ${ }^{2}$ For example, some metastatic melanoma patients who were treated with ipilimumab or nivolumab experienced long-term complete remission. ${ }^{2,19}$ In the KEYNOTE-006 pivotal trial that led to the approval of pembrolizumab by the FDA, $96.7 \%$ of the patients who responded had sustained responses after a median follow-up of 7.9 months. ${ }^{20}$ Therefore, immunotherapy trials often use progression-free survival (PFS) as the efficacy endpoint rather than a simple binary outcome indicating tumor response or no response.

Moreover, immune response is a unique and important outcome for immunotherapy, 
which measures the biological efficacy of the immunotherapeutic agent in activating the immune system. The immune response is agent-specific, including the proliferation of CD8+ T-cells, CD4+ T-cells and various cytokines, e.g., IFN- $\alpha$, IL-1 $\beta$, IL-6, IL-8. For each patient, the immune response is typically measured repeatedly over time in a longitudinal setting. Since immunotherapy achieves its treatment effect by activating a patient's immune system, the immune response is typically closely associated with the treatment effect of immunotherapy, and previous studies have confirmed this. ${ }^{21-27}$ The pattern and magnitude of the immune response are an important indicator of the disease progression. The cured group and susceptible group may exhibit different patterns of the immune response over time, so the trajectory of the immune response can be predictive of the efficacy outcome for a censored patient. Thus it is critical to incorporate the longitudinal immune response in the trial design and leverage its close relationship with efficacy for decision making.

Our research is motivated by a two-arm phase II clinical trial which is being conducted at the Indiana University Melvin and Bren Simon Cancer Center. The objective of the trial is to compare the effectiveness of two immunotherapeutic agents A and B for treating patients with recurrent platinum-resistant epithelial ovarian cancer, where A is a "standard" immunotherapeutic agent that has already been approved by FDA, and B is a novel experimental immunotherapeutic agent to be tested against A. Both agents act against the programmed cell death ligand 1 (PD-L1) checkpoint inhibitor in recurrent ovarian cancer. A maximum of 80 patients will be accrued to the trial. Immune response is measured by the count of CD8+ T-cells, which will be measured at baseline and at 2-month intervals over a 12-month follow-up period. The efficacy endpoint is the progression free survival (PFS). The primary objective of the trial is to assess if the experimental agent is more efficacious than the standard agent in terms of the immune response and PFS.

To capture the unique features of immunotherapy, we propose a novel Bayesian phase II randomized design for immunotherapy trials that jointly models the longitudinal immune response and time-to-event efficacy (BILITE). To accommodate the fact that some patients 
may have long-term durable responses, we assume a fraction of patients are "cured" by the treatment. The longitudinal immune response is modeled using hierarchical nonlinear mixed-effects models with different models for the cured and susceptible groups; and conditional on the immune response trajectory, the time-to-event efficacy data for patients in the susceptible group is modeled via a time-dependent Cox-type regression model. We quantify the desirability of the treatment using a utility function elicited from physicians. A two-stage design is proposed to assign new patients to desirable treatment through adaptive randomization. Joint modeling of longitudinal measurements and survival outcome has been considered in the literature. ${ }^{28-37}$ In this article, we adapt these methods to accommodate the unique features of immunotherapy. To the best of our knowledge, this article provides the first phase II design for immunotherapy that jointly accounts for longitudinal immune response and time-to-event efficacy.

The remainder of this article is organized as follows. In Section 2, we describe the probability models for the longitudinal immune response and the time-to-event efficacy outcome, and present the two-stage algorithm. In Section 3, we examine the operating characteristics of the BILITE design through simulation studies. We provide concluding remarks in Section 4.

\section{Methods}

\subsection{Probability models}

Consider a 2-arm phase II trial comparing a new immunotherapeutic agent with a standard immunotherapeutic agent. Let $Z$ denote the treatment index, with $Z=0$ or 1 if a patient receives the standard or the experimental treatment, respectively. Due to the fact that some patients may achieve durable response (i.e., be "cured"), there are two distinct subgroups of patients, those that are cured and those that are susceptible to disease progression. Let $X_{i}$ be the patient susceptible status index with $X_{i}=0$ or 1 if patient $i$ is cured or susceptible 
to disease progression. $X_{i}$ is a partially observed variable. If patient $i$ experiences disease progression, then $X_{i}=1$; however, if patient $i$ does not experience disease progression due to censoring, the value of $X_{i}$ is unknown. For $z=0,1$, let $\pi_{z}=\operatorname{Pr}\left(X_{i}=1 \mid Z_{i}=z\right)$ be the probability of being a susceptible patient treated with agent $Z=z$.

We model the longitudinal immune response using hierarchical nonlinear mixed-effects models. The immune response $M$ is taken to be the increase of an immune activity from baseline to some measurement time post treatment, which is generally a non-negative continuous outcome. $M$ is measured repeatedly after the initiation of the treatment. Typically the immune response is scheduled to be measured at pre-specified time points during patients' regular check-up visits, say every month or every 2 months. We do not require it to be measured at each scheduled visit for every patient, but expect most patients to be able to provide measurements at each scheduled visit. For patient $i$, let $\mathbf{M}_{i}=\left(M_{i 1}, \cdots, M_{i J}\right)$ denote the set of longitudinal measurements of the immune response, where $M_{i j}$ denotes the observed immune response measured at time $t_{i j}$ and $J$ is the number of scheduled measurements for the immune response in the follow-up window, $j=1, \cdots, J$. Here for notational brevity, we use the same notation $J$ to denote the number of immune response measurements for all patients, but our method allows different patients to have different numbers of measurements taken on different time schedules.

Letting $M_{i j}^{*}$ denote the true immune response for patient $i$ at time $t_{i j}$, we assume

$$
M_{i j}=M_{i j}^{*}+\epsilon_{i j}, j=1, \cdots, J
$$

where $\epsilon_{i j} \stackrel{i i d}{\sim} N\left(0, \sigma^{2}\right)$ is the error term.

The true immune response trajectory for a patient may take various shapes, including increasing, first-increasing-then-decreasing, and first-increasing-then-plateauing, so we use a flexible trajectory model that can accommodate all these possibilities. Let $M_{i}^{*}(t)$ be the function of the true immune response for patient $i$ at time $t$, which is determined by 
parameters $\alpha_{i}, \eta_{0 i}, \eta_{1 i}$, and $\eta_{2 i}$ for patient $i$. We model $M \quad{ }_{i}^{*}(t)$ as

$$
\log \left(\frac{M_{i}^{*}(t)}{\alpha_{i}-M_{i}^{*}(t)}\right)=\eta_{0 i}+\eta_{1 i} t+\eta_{2 i} t^{2}
$$

In this model, $\alpha_{i}$ is the maximum possible immune response so $\alpha_{i}>M_{i}^{*}(t)>0$ for all $t>0, \eta_{1 i}$ and $\eta_{2 i}$ characterize the shape of the immune response trajectory over time. Since $M_{i}^{*}(t)$ represents the pre-post difference in the immune activity, it is expected to be 0 when $t=0$.

So we set $\eta_{0 i}=-3$ so that $M{ }_{i}^{*}(0)$ is virtually 0 at $t=0$. It follows directly that

$$
M_{i}^{*}(t)=\frac{\left.\underline{\alpha}_{i} \underline{\exp \left(\eta_{0 i}+\eta_{1 i}\right.} \underline{t}+\eta_{2 i} \underline{t} \underline{\underline{t}}\right) 1}{+\exp \left(\eta_{0 i}+\eta_{1 i} t+\eta_{2 i} t^{2}\right)}
$$

This model can accommodate increasing, first-increasing-then-plateauing, and first-increasingthen-decreasing patterns of the immune response in the follow-up window $\left(0, t_{\text {max }}\right)$. Figure 1 shows 3 trajectories in the 12-month follow-up window generated from this model. In particular, the first-increasing-and-then-plateauing shape in the follow-up window $\left(0, t_{\max }\right)$

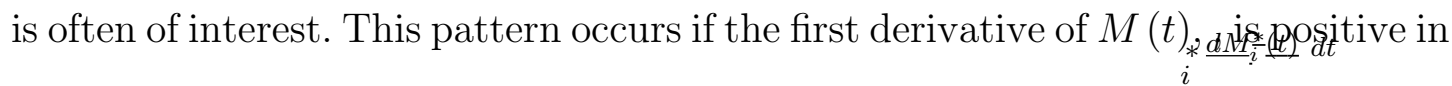
the range $\left(0, t_{\max }\right)$ and $M_{i}^{*}\left(t_{\max }\right)$ is very close to $\alpha_{i}$. More precisely, the condition is

- $\eta_{1 i}+2 \eta_{2 i} t>0$ for $0<t<t_{\max }$

- $\exp \left(\eta_{0 i}+\eta_{1 i} t_{\max }+\eta_{2 i} t_{\max }^{2}\right)$ $\overrightarrow{1+\exp \left(\eta_{0 i}+\eta_{1 i} t_{\max }+\eta_{2 i} t_{\text {max }}^{2}\right)}$ is very close to 1 , say, 0.999 .

The level of plateauing is $\alpha_{i}$.

We decompose $\alpha_{i}, \eta_{1 i}$ and $\eta_{2 i}$ into fixed and random effects. Since the immune response trajectory may exhibit different patterns for patients who experience disease progression and patients who are cured, we use different decomposition parameters for patients in the two groups. If patient $i$ is cured (i.e., $\left.X_{i}=0\right), \alpha_{i}, \eta_{1 i}$ and $\eta_{2 i}$ are decomposed as

$$
\eta_{1 i}=\beta_{1}+\beta_{2} Z_{i}+b_{i 1}
$$




$$
\begin{gathered}
\eta_{2 i}=\beta_{3}+\beta_{4} Z_{i}+b_{i 2} \\
\log \left(\alpha_{i}\right)=\beta_{5}+\beta_{6} Z_{i}+b_{i 3}
\end{gathered}
$$

where $Z_{i}=0$ or 1 if patient $i$ receives the standard treatment or the experimental treatment, respectively, $\boldsymbol{\beta}=\left(\beta_{1}, \beta_{2}, \beta_{3}, \beta_{4}, \beta_{5}, \beta_{6}\right)$ contains fixed effects, and the vector of random effects $\boldsymbol{b}_{i}=\left(b_{i 1}, b_{i 2}, b_{i 3}\right)$ follows a tri-variate normal distribution with mean 0 and covariance matrix $\Sigma_{0}$. Log transformations of $\alpha_{i}$ are used to guarantee that they are positive. Letting $\boldsymbol{\phi}_{i}=$ $\left(\eta_{1 i}, \eta_{2 i}, \log \left(\alpha_{i}\right)\right),(4),(5)$, and (6) are equivalent to

$$
\boldsymbol{\phi}_{i} \mid\left\{X_{i}=0, Z_{i}\right\} \sim N_{3}\left(\tilde{Z}_{i} \boldsymbol{\beta}, \Sigma_{0}\right)
$$

where $Z_{i}^{*}=c\left(1, Z_{i}\right)$ and $\tilde{Z}_{i}=\left(I_{3} \otimes Z_{i}^{*}\right)^{T}, N_{3}\left(\tilde{Z}_{i} \boldsymbol{\beta}, \Sigma_{0}\right)$ denotes a trivariate normal distribution with mean vector $\tilde{Z}_{i} \boldsymbol{\beta}$ and covariance matrix $\Sigma_{0}$.

For a susceptible patient $i$ with $X_{i}=1$, the same decomposition is assumed with a different set of parameters to accommodate possibly different trajectories for patients in the two subgroups:

$$
\phi_{i} \mid\left\{X_{i}=1, Z_{i}\right\} \sim N_{3}\left(\tilde{Z}_{i} \gamma, \Sigma_{1}\right)
$$

where $\gamma=c\left(\gamma_{1}, \gamma_{2}, \gamma_{3}, \gamma_{4}, \gamma_{5}, \gamma_{6}\right)$ and $\Sigma_{1}$ is a $3 \times 3$ covariance matrix. With different decomposition parameters for patients in the cured group and the susceptible group, the treatment is allowed to influence the trajectories of the immune response in different ways in the two subgroups. Due to the typical small sample sizes of phase II trials, we let $\Sigma_{0}=\Sigma_{1} \equiv \Sigma$ in this article to obtain a more parsimonious model.

Now let's turn to the distribution of PFS conditional on the immune response. Let $S\left(t \mid Z_{i}, \phi_{i}\right)$ denote the survival function of PFS for patient $i$ with random effects $\boldsymbol{\phi}_{i}$ who is treated with agent $Z_{i}$. To accommodate the fact that a certain percentage of the patients may be "cured", we use the following cure rate model to model the time to disease progression 
$T$,

$$
S\left(t \mid Z_{i}, \phi_{i}\right)=\left(1-\pi_{Z_{i}}\right)+\pi_{Z_{i}} S_{u}\left(t \mid Z_{i}, \phi_{i}\right)
$$

where $S_{u}\left(t \mid Z_{i}, \phi_{i}\right)$ is the survival function for patients who are susceptible to disease progression.

For a susceptible patient $i$, conditional on the random effects $\phi_{i}$, we model $S_{u}\left(t \mid Z_{i}, \phi_{i}\right)$ using a time-dependent Cox-type regression model with baseline hazard following the Weibull distribution,

$h_{u}\left(t \mid Z_{i}, \phi_{i}\right)=\lambda \kappa t^{\kappa-1} \exp \left\{\gamma_{s 1} M_{i}^{*}(t)+\gamma_{s 2} Z_{i}\right\}=\lambda \kappa t^{\kappa-1} \exp \left\{\gamma_{s 1} \frac{\alpha_{i} \exp \left(\eta_{0 i}+\eta_{1 i} t+\eta_{2 i} t^{2}\right)}{1+\exp \left(\eta_{0 i}+\eta_{1 i} t+\eta_{2 i} t^{2}\right)}+\gamma_{s 2} Z_{i}\right\}$

where $\lambda$ and $\kappa$ are the scale and shape parameters of the baseline Weibull distribution, respectively, $M_{i}^{*}(t)$ is the true immune response for patient $i$ at time $t, \gamma_{s 2}$ represents the direct effect of the treatment on the hazard, and $\gamma_{s 1}$ represents the indirect effect of the treatment on the hazard through the immune response. Since immunotherapy achieves its therapeutic effect of killing cancer cells by activating the immune response, higher values of the immune response are generally considered more desirable than lower values, because they potentially translate into better treatment efficacy. So we restrict $\gamma_{s 1}$ to be negative, i.e., $\gamma_{s 1}<0$. We take this parametric approach with a Weibull baseline hazard, rather than the semiparametric approach that does not specify the baseline hazard, because of the limited sample size of early phase trials. Previous studies ${ }^{38,39}$ and the sensitivity analysis described later show that for the purpose of treatment comparison, our design is not sensitive to this parametric assumption. As a side note, this model has a similar form as the Cox proportional hazards model, but the hazard ratio may vary over time due to the time-dependent covariate $M_{i}^{*}(t)$

The corresponding survival function is

$$
S_{u}\left(t \mid Z_{i}, \phi_{i}\right)=\exp \left[-\int_{0}^{t} \lambda \kappa u^{\kappa-1} \exp \left\{\gamma_{s 1} M_{i}^{*}(u)+\gamma_{s 2} Z_{i}\right\} d u\right]
$$




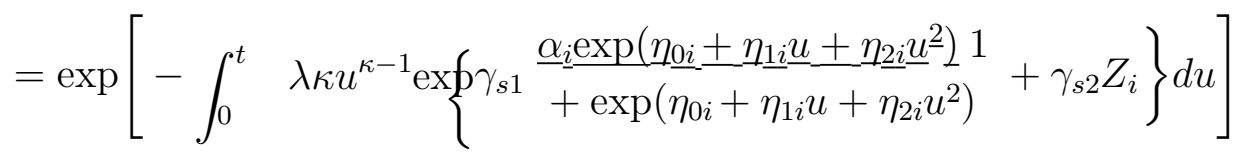

For the $i$ th patient, let $c_{i}$ denote the censoring time, $y_{i}=\min \left(t_{i}, c_{i}\right)$ denote the actual observed time, and $\delta_{i}=I\left(t_{i} \leq c_{i}\right)$ be the censoring indicator. Then the observed data from the $i$ th patient are $\mathcal{D}_{i}=\left(Z_{i}, \mathbf{M}_{i}, y_{i}, \delta_{i}\right)$, and the missing or latent data are $\left(X_{i}, \phi_{i}\right)$. Letting $\Theta=\left(\sigma^{2}, \boldsymbol{\beta}, \boldsymbol{\gamma}, \Sigma, \pi_{0}, \pi_{1}, \lambda, \kappa, \gamma_{s 1}, \gamma_{s 2}\right)$ represent all parameters, the complete data likelihood for the $i$ th patient is

$$
\begin{aligned}
L_{i}(\Theta)= & \left\{p\left(\mathbf{M}_{i} \mid \boldsymbol{\phi}_{i}, \sigma^{2}\right) p\left(\boldsymbol{\phi}_{i} \mid \boldsymbol{\beta}, \Sigma\right)\left(1-\pi_{Z_{i}}\right)\right\}^{I\left(X_{i}=0\right) I\left(\delta_{i}=0\right)} \\
\times & \left\{f_{u}\left(y_{i} \mid Z_{i}, \boldsymbol{\phi}_{i}\right)^{I\left(\delta_{i}=1\right)} S_{u}\left(y_{i} \mid Z_{i}, \boldsymbol{\phi}_{i}\right)^{I\left(\delta_{i}=0\right)} p\left(\mathbf{M}_{i} \mid \boldsymbol{\phi}_{i}, \sigma^{2}\right) p\left(\boldsymbol{\phi}_{i} \mid \boldsymbol{\gamma}, \Sigma\right) \pi_{Z_{i}}\right\}^{I\left(X_{i}=1\right)}
\end{aligned}
$$

where $p\left(\mathbf{M}_{i} \mid \boldsymbol{\phi}_{i}, \sigma^{2}\right)$ is the density for the longitudinal immune data $\mathbf{M}_{i}$ :

$$
p\left(\mathbf{M}_{i} \mid \phi_{i}, \sigma^{2}\right)=\prod_{j=1}^{I_{i}} \phi\left\{M_{i j} ; M_{i j}^{*}, \sigma^{2}\right\}
$$

Here $\phi\left(. ; \mu, \sigma^{2}\right)$ is the density of a normal distribution with mean $\mu$ and variance $\sigma^{2}$, and $I_{i}$ is the number of post-treatment immune responses that have been measured for patient $i$ at an interim decision time. Note that $I_{i}$ may not equal $J$ because due to the different entry times of patients, different patients may have different numbers of observed immune response at an interim decision time.

Letting $\mathcal{D}_{n}=\left\{\mathcal{D}_{i}, i=1, \cdots, n\right\}$ denote the data from the $n$ patients when an interim decision is to be made, the likelihood based on $n$ patients is $L(\Theta) \propto \quad \prod_{i=1}^{n} L_{i}(\Theta)$.

Letting $f(\Theta)$ denote the joint prior distribution of $\Theta$ (which will be described in detail in the next section), the joint posterior distribution of $\Theta$ based on data from the first $n$ patients in the trial is $f\left(\Theta \mid \mathcal{D}_{n}\right) \propto f(\Theta) L\left(\Theta ; \mathcal{D}_{n}\right)$. We sample this posterior distribution using the Gibbs sampler, ${ }^{40,41}$ which has the advantage of having posterior draws for all parameters, including the latent variables $X_{i}$ and $\boldsymbol{\phi}_{i}$. 


\subsection{Prior specification}

To specify the prior distributions for the hyperparameters $\beta_{1}, \beta_{2}, \beta_{3}, \beta_{4}, \gamma_{1}, \gamma_{2}, \gamma_{3}, \gamma_{4}$, we follow the idea of Gelman et al.. ${ }^{42}$ Note that equation 22 is equivalent to $\log \left(\frac{M_{i}^{*}(t) / \alpha_{i}}{1-M_{i}^{*}(t) / \alpha_{i}}\right)=\eta_{0 i}+$ $\eta_{1 i} t+\eta_{2 i} t^{2}$, which has the standard logistic regression form given that the range of $M_{i}^{*}(t)$ is $\left(0, \alpha_{i}\right)$. A change of 5 moves $M_{i}^{*}(t) / \alpha_{i}$ from 0.01 to 0.5 or from 0.5 to 0.99 , which is considered unlikely for a typical change in a covariate. Therefore, after scaling the input variable $t$ to have standard deviation $0.5, \eta_{1}$ and $\eta_{2}$ should be mostly between -5 and 5 so that a change in $t$ from one standard deviation below the mean to one standard deviation above the mean will most likely result in a difference of less than 5 for the regression in equation (2). Since $\beta_{1}$ and $\beta_{1}+\beta_{2}$ are the mean values of $\eta_{i 1}$ for treatment arms 0 and 1 , respectively, we assign $\beta_{1}$ and $\beta_{2}$ normal prior distributions $\beta_{1} \sim N\left(0,2.5^{2}\right)$ and $\beta_{2} \sim N\left(0,5^{2}\right)$ so that a priori, there is $95 \%$ chance that $\beta_{1}$ is between -5 and 5 , and for any value of $\beta_{1}$ in that range, $\beta_{1}+\beta_{2}$ is mostly between -5 and 5 . Likewise, $\beta_{3}, \gamma_{1}$, and $\gamma_{3}$ are assigned normal prior distributions $N\left(0,2.5^{2}\right)$ and $\beta_{4}, \gamma_{2}$, and $\gamma_{4}$ are assigned normal prior distributions $N\left(0,5^{2}\right)$.

Since $\alpha_{i}$ has the interpretation of the maximum immune response, we elicit the following information from physicians: for cured patients, elicit the ranges of the maximum immune response $\alpha$ for the two treatments, denoted as $\left(\hat{\alpha}_{0 L}^{C}, \hat{\alpha}_{0 U}^{C}\right)$ for treatment 0 and $\left(\hat{\alpha}_{1 L}^{C}, \hat{\alpha}_{1 U}^{C}\right)$ for treatment 1 . So $\log (\alpha)$ is between $\left(\log \left(\hat{\alpha}_{0 L}^{C}\right), \log \left(\hat{\alpha}_{0 U}^{C}\right)\right)$ for treatment 0 and $\left(\log \left(\hat{\alpha}_{1 L}^{C}\right), \log \left(\hat{\alpha}_{1 U}^{C}\right)\right)$ for treatment 1. So $\beta_{5} \in\left(\log \left(\hat{\alpha}_{0 L}^{C}\right), \log \left(\hat{\alpha}_{0 U}^{C}\right)\right)$, and $\beta_{6} \in\left(\log \left(\hat{\alpha}_{1 L}^{C}\right)-\right.$ $\left.\log \left(\hat{\alpha}_{0 U}^{C}\right), \log \left(\hat{\alpha}_{1 U}^{C}\right)-\log \left(\hat{\alpha}_{0 L}^{C}\right)\right)$. Therefore, we assign each of them a normal prior centered at the interval mean and standard deviation $1 / 4$ of the interval range so that with probability $95 \%$ these two parameters are in the above ranges. Prior distributions for $\gamma_{5}$ and $\gamma_{6}$ can be obtained similarly by eliciting the ranges of $\alpha$ for susceptible patients: $\left(\hat{\alpha}_{0 L}^{S}, \hat{\alpha}_{0 U}^{S}\right)$ for treatment 0 and $\left(\hat{\alpha}_{1 L}^{S}, \hat{\alpha}_{1 U}^{S}\right)$ for treatment 1 . For example, for our motivating trial, these elicited ranges were $\left(\hat{\alpha}_{0 L}^{C}, \hat{\alpha}_{0 U}^{C}\right)=(15,25),\left(\hat{\alpha}_{1 L}^{C}, \hat{\alpha}_{1 U}^{C}\right)=(25,35),\left(\hat{\alpha}_{0 L}^{S}, \hat{\alpha}_{0 U}^{S}\right)=(5,15),\left(\hat{\alpha}_{1 L}^{S}, \hat{\alpha}_{1 U}^{S}\right)=(5,15)$. So we set $\beta_{5} \sim N\left(2.95,0.125^{2}\right), \beta_{6} \sim N\left(0.425,0.21^{2}\right), \gamma_{5} \sim N\left(2.15,0.275^{2}\right), \gamma_{6} \sim N\left(0,0.55^{2}\right)$. 
The covariance matrix $\Sigma$ for the random effects $\boldsymbol{b}_{i}$ is assigned an inverse Wishart prior distribution $\Sigma \sim \operatorname{Inv-Wishart}\left(\nu_{0}, \Lambda_{0}\right)$ where we take $\Lambda_{0}$ to be a diagonal matrix with diagonal elements $\varphi_{1}, \varphi_{2}, \varphi_{3}$ :

$$
\Lambda_{0}=\left[\begin{array}{ccc}
\varphi_{1} & 0 & 0 \\
0 & \varphi_{2} & 0 \\
0 & 0 & \varphi_{3}
\end{array}\right]
$$

To specify $\nu_{0}$ and $\Lambda_{0}$, note the three diagonal elements of $\Sigma$ (denoted as $\sigma_{1}^{2}, \sigma_{2}^{2}$, and $\sigma_{3}^{2}$ ) are the variances of $\eta_{1 i}, \eta_{2 i}$, and $\log \left(\alpha_{i}\right) . \eta_{1 i}$ and $\eta_{2 i}$ are mostly between -5 and 5 when $t$ is standardized, so $\sigma_{1}^{2}$ and $\sigma_{2}^{2}$ should be mostly between 0 and 25 , so that $2 \sigma_{1}\left(2 \sigma_{2}\right)$ can cover most of the range of $\eta_{1}\left(\eta_{2}\right)$. The range of $\log \left(\alpha_{i}\right)$ can obtained from the elicited values from physicians. For example, based on the elicited ranges of $\alpha$ for our motivating trial described in the previous paragraph, the largest range of $\log \left(\alpha_{i}\right)$ is $(\log (5), \log (15))=(1.6,2.7)$. So $\sigma_{3}^{2}$ should be mostly between 0 and $0.55^{2}$. We set $\nu_{0}=14, \varphi_{1}=\varphi_{2}=125$, and $\varphi_{3}=1.5$ so that for each diagonal element of $\Sigma$, twice of the standard deviations of the mean covers the above range of the variance.

We assign $\sigma^{2}$ a vague inverse Gamma prior distribution, e.g., $\sigma^{2} \sim I G(0.1,0.1)$, so that the data will dominate the posterior distribution. $\lambda$ and $\kappa$ are assigned vague Gamma distributions, e.g., $\lambda \sim \operatorname{Gamma}(0.1,0.1), \kappa \sim \operatorname{Gamma}(0.1,0.1) . \quad \pi_{0}$ and $\pi_{1}$ are assigned Unif $(0,1)$ prior distributions.

The elicitation of the prior for $\gamma_{s 1}$ in the hazard function (10) is facilitated by the fact that it is the log of the hazard ratio when the immune response increases by 1 . We elicit from clinicians a range of hazard ratio that is practically feasible, denoted as $\left(r_{1 L}, r_{1 U}\right)$, and then assign $\gamma_{s 1}$ a uniform prior distribution with range $\left(\log \left(r_{1 L}\right), \log \left(r_{1 U}\right)\right)$. The elicitation of the prior for $\gamma_{s 2}$ is analogous as $\exp \left(\gamma_{s 2}\right)$ represents the hazard ratio of PFS between the two treatments. We elicit from physicians a range of the hazard ratio that is practically feasible, say $\left(r_{2 L}, r_{2 U}\right)$, and then assign $\gamma_{s 2}$ a uniform prior distribution $\gamma_{s 2} \sim \operatorname{Unif}\left(\log \left(r_{2 L}\right), \log \left(r_{2 U}\right)\right)$. 


\subsection{Decision Criteria}

We propose to make decisions based on a utility function elicited from physicians. Let $t_{\max }$ denote the maximum follow-up time for patients. To facilitate the elicitation of the utility, we partition the support of $T$ into $L$ intervals, $\left(\xi_{1}, \xi_{2}\right], \cdots,\left(\xi_{L-1}, \xi_{L}\right],\left(\xi_{L}, \xi_{L+1}\right]$, where $\xi_{1} \equiv 0$, $\xi_{L} \equiv t_{\max }$ and $\xi_{L+1} \equiv \infty$. For example, in the motivating trial, the maximum follow-up time $t_{\text {max }}$ is 12 months, and the support of $T$ is partitioned into 5 intervals $(0,3],(3,6],(6,9],(9$, 12], $(12, \infty]$. Let $\zeta\left(\xi_{l}, \xi_{l+1}\right]$ denote the utility for the outcome $\left(T \in\left(\xi_{l}, \xi_{l+1}\right]\right), l=1, \cdots, L$, and $\zeta$ (Cured) denote the utility for the "cured" outcome. Using the motivating trial as an example, utility $\zeta$ is elicited from physicians as follows: fix the desirability of the most desirable outcome (i.e., Cured) as $\zeta$ (Cured) $=100$ and the desirability of the least desirable outcome $(T \in(0,3])$ as $\zeta(0,3]=0$, and then ask physicians to use these two pairs as the reference for scoring the desirability of the other categories using the scale of $(0,100)$. Table 1 shows the elicited utility for the motivating trial.

Based on the elicited utility function, the true utility for treatment $Z$ is defined as

$$
U_{\text {true }}(Z)=\sum_{l=1}^{L} \zeta\left(\xi_{l}, \xi_{l+1}\right] \operatorname{Pr}\left(T \in\left(\xi_{l}, \xi_{l+1}\right] \mid Z, \Theta\right)+\zeta(\text { Cured }) \operatorname{Pr}(T=\infty \mid Z, \Theta) .
$$

where $\operatorname{Pr}\left(T \in\left(\xi_{l}, \xi_{l+1}\right] \mid Z, \Theta\right)$ is obtained by integrating over the random effects $\phi_{i}=$ $\left(\eta_{1 i}, \eta_{2 i}, \log \left(\alpha_{i}\right)\right)$. The experimental treatment is considered promising compared with the standard treatment if the increase in the true utility exceeds a certain percentage $\Delta_{0}$, that is,

$$
\Delta_{\text {true }}=\frac{U_{\text {true }}(Z=1)-U_{\text {true }}(Z=0)}{U_{\text {true }}(Z=0)}>\Delta_{0},
$$

where $\Delta_{0}$ is a minimum meaningful increment in utility pre-specified by clinicians.

During the trial, given the interim data $\mathcal{D}_{n}$ from the first $n$ patients, we evaluate the 
desirability of treatment $Z$ based on the posterior estimate of its utility,

$$
U_{n}(Z)=\sum_{l=1}^{L} \zeta\left(\xi_{l}, \xi_{l+1}\right] \operatorname{Pr}\left(T \in\left(\xi_{l}, \xi_{l+1}\right] \mid Z, \mathcal{D}_{n}\right)+\zeta(\text { Cured }) \operatorname{Pr}\left(T=\infty \mid Z, \mathcal{D}_{n}\right)
$$

The posterior estimate of the percentage of increase in utility $\Delta_{\text {true }}$ is obtained as

$$
\Delta_{n}=\frac{U_{n}(Z=1)-U_{n}(Z=0)}{U_{n}(Z=0)}
$$

For a clinical trial with both longitudinal immune response and time-to-event efficacy data, decision making is difficult at the beginning of the trial because of the highly unstable estimates of the survival curves due to the small number of patients and possibly incomplete immune response and/or censored efficacy data. To alleviate this issue, we take a twostage design. Let the maximum sample size be $N$. In stage I, we enroll $N_{1}$ patients and equally randomize them to the two treatment arms. The purpose of this stage is to collect preliminary data to facilitate the model fitting in stage II. We require that patients accrued during stage I be completely followed before stage II model-based algorithm is in effect. In stage II, we continue to enroll the remaining $N_{2}=N-N_{1}$ patients. Assuming that $n$ patients have been enrolled in the trial,

1. If $\operatorname{Pr}\left(\Delta_{n}>\Delta_{0}\right)>c_{1}$, the trial is terminated and treatment $Z=1$ is claimed to be superior to $Z=0$.

2. If $\operatorname{Pr}\left(\Delta_{n}>\Delta_{0}\right)<c_{2}$, the trial is terminated and treatment $Z=1$ is claimed to be futile.

3. Otherwise, adaptively randomize the next cohort of patients to the two treatments $Z=1$ and $Z=0$ with probabilities $\operatorname{Pr}\left(\Delta_{n}>\Delta_{0}\right)$ and $\operatorname{Pr}\left(\Delta_{n} \leq \Delta_{0}\right)$, respectively.

4. Repeat steps 1-3 until reaching the maximum sample size or terminating the trial early.

If the maximum number of patients $N$ is reached and neither superiority nor futility has been 
claimed, we claim superiority of treatment $Z=1$ if $\operatorname{Pr}\left(\Delta_{n}>\Delta_{0}\right)>c_{0}$ and claim futility otherwise. In the algorithm, $c_{1}, c_{2}$ and $c_{0}$ are probability cutoffs that will be tuned through simulation studies to achieve desirable design operating characteristics, e.g., the selection percentage of each treatment arm and the average number of patients in each arm.

\section{Simulation}

We conducted simulation studies to assess the operating characteristics of the proposed BILITE design. Taking the setting of the motivating trial, the maximum sample size $N=80$ and stage I sample size $N_{1}=30$. Patients were treated in cohorts of size 5 . The follow-up time for patients was $t_{\max }=12$ months, and patient accrual followed a Poisson process with a rate of 3 per month. We used the utility function in Table 1. The minimum meaningful improvement of the utility was taken to be $\Delta_{0}=30 \%$ so that the experimental agent was deemed promising relative to the standard treatment if the increase in utility exceeded $30 \%$. To reflect the elicited information from clinicians, the hyperprior distributions for $\beta_{5}, \beta_{6}, \gamma_{5}, \gamma_{6}$ were specified as $\beta_{5} \sim N\left(2.95,0.125^{2}\right), \beta_{6} \sim N\left(0.425,0.21^{2}\right), \gamma_{5} \sim N\left(2.15,0.275^{2}\right), \gamma_{6} \sim$ $N\left(0,0.55^{2}\right)$, and the covariance matrix $\Sigma \sim \operatorname{Inv}$-Wishart $\left(14, \Lambda_{0}\right)$ where $\Lambda_{0}$ is a diagonal matrix with diagonal elements 125,125 , and 1.5. We set the prior distribution of $\gamma_{s 1}$ and $\gamma_{s 2}$ as $\gamma_{s 1} \sim U(-1.6,0)$ and $\gamma_{s 2} \sim U(-0.4,0.4)$ to reflect the prior knowledge from clinicians that the hazard ratio when the immune response increases by 1 was between 0.2 and 1 , and the hazard ratio of PFS between the two treatments was between 0.7 and $1 / 0.7$. We took the probability cutoffs $c_{1}=0.9, c_{2}=0.1$, and $c_{0}=0.58$, which were calibrated to achieve overall desirable operating characteristics.

We compared the BILITE design with two alternative designs. One is a "more conven-

tional" phase II (CPT) design that ignores the immune response. To make the two designs comparable, the same PFS model was used in the CPT design, but with the term involving 
immune response dropped. More precisely, for the CPT design, the PFS model was

$$
S\left(t \mid Z_{i}\right)=\left(1-\pi_{Z_{i}}\right)+\pi_{Z_{i}} S_{u}\left(t \mid Z_{i}\right)
$$

where

$$
h_{u}\left(t \mid Z_{i}\right)=\lambda \kappa t^{\kappa-1} \exp \left\{\gamma_{s 2} Z_{i}\right\}
$$

So basically the only difference between BILITE and CPT designs is that BILITE takes into account the immune response while $\mathrm{CPT}$ does not. The difference in the performance between the two designs would demonstrate the importance of incorporating the immune response in immunotherapy trials. The second design is a conventional phase II design that incorporated the observed immune response measurements, which is denoted as CPTO design. The CPTO design utilized the same model as the proposed design, but with $M_{i}^{*}(t)$ replaced by $\bar{M}_{i}$, the average of the measured values $M_{i j}$ for patient $i$ at an interim decision time, that is, the PFS model was

$$
S\left(t \mid Z_{i}\right)=\left(1-\pi_{Z_{i}}\right)+\pi_{Z_{i}} S_{u}\left(t \mid Z_{i}\right)
$$

where

$$
h_{u}\left(t \mid Z_{i}\right)=\lambda \kappa t^{\kappa-1} \exp ^{\left\{\gamma_{s 1} \bar{M}_{i}+\gamma_{s 2} Z_{i}\right\}}
$$

The design algorithm is the same for the three designs. Under each scenario, we simulated 1000 trials.

We designed 9 scenarios that cover different situations of the immune response and PFS. The true regression parameters for the 9 scenarios are provided in Table S1 in the Supplementary Materials. Data sharing is not applicable to this paper as all data in this article is computer simulated. In the first 7 scenarios, the experimental treatment is superior to the standard treatment, and in the last 2 scenarios, the experimental treatment is not promising. Figure 2 shows the immune response trajectory for the cured and susceptible subgroups 
under the two treatment arms in each scenario when the random effects $\boldsymbol{b}_{i}$ take on their mean values 0 . As Figure 2 shows, the 9 scenarios covered different situations of the im-mune trajectories. For example, in scenario 1, the immune response trajectory shows a first increasing and then plateaued trend for both subgroups under either treatments. In sce-nario 3, the immune response increases over time for the cured subgroup and first increases and then plateaus for the susceptible subgroup under both treatments. In scenario 5, the immune response first increases and then decreases for the susceptible subgroup under the experimental treatment and increases under the standard treatment.

Table 2 summarizes the operating characteristics of our proposed BILITE design, the CPT design, and the CPTO design. Under each scenario, we report the selection percentage of each treatment, the average number of patients and average number of patients in stage II with adaptive randomization under each treatment, and the total number of patients. In scenarios 1-7, the experimental treatment is superior to the standard treatment. The BILITE design yielded better or comparable performance than the CPT and CPTO designs. For example, in scenario $1, \Delta_{\text {true }}=0.52$. The three designs gave similar selection percentages of the experimental treatment (65.9\% under BILITE, 61.5\% under CPT, and 65.3\% under CPTO), and BILITE assigned slightly higher number of patients to the experimental arm in stage II (19.7 vs 18.1 and 18.2). In scenario $3, \Delta_{\text {true }}=0.48$. The BILITE design selected the experimental treatment $58.8 \%$ of the time and assigned 17.8 patients to the experimental treatment in stage II, while the CPT (CPTO) design selected it 48.9\% (49.4\%) of the time and allocated 19.9 (17.8) patients in stage II. In scenario $6, \Delta_{\text {true }}=0.67$. The BILITE design resulted in more than $15 \%$ higher correct treatment selection than the CPT and CPTO designs (i.e., $73.2 \%$ vs $56.6 \%$ and $52.8 \%$ ), and the three designs assigned comparable number of patients in stage II $(18.3,18.1$, and 18.7). In scenarios 8 and 9 , the experimental arm is futile. The BILITE design correctly claimed futility about $80 \%$ of the time $(80.6 \%$ and $77.7 \%$ under scenarios 8 and 9, respectively). The CPT and CPTO designs yielded similar or worse selection rates than the BILITE design. 


\subsection{Sensitivity analyses}

We carried out sensitivity analyses to examine the robustness of our proposed design by using 1) a smaller sample size, 2) another set of utility values, 3) different prior distributions, and 4) a log-logistic baseline hazard for patients who are susceptible to disease progression.

When the maximum sample size dropped from 80 to 60 , the performance of the BILITE design was slightly worse but still satisfactory, as summarized in Figure 3 for scenarios 1-6. Since the maximum sample size differed, we reported in Figure 3 the percentage of patients in stage II who were randomized to the experimental treatment.

We considered an alternative utility function displayed in Table 3, which gave higher desirability scores to the non-cured categories. As shown in Figure 3, the results under the alternative utility are generally similar to the original results, suggesting that the BILITE design is robust to the elicited utility.

We evaluated the sensitivity of the BILITE design to different prior distributions. We made all the priors more non-informative. Specifically, we assigned normal priors $N\left(0,5^{2}\right)$ to $\beta_{1}, \beta_{3}, \gamma_{1}, \gamma_{3}$, normal priors $N\left(0,10^{2}\right)$ to $\beta_{2}, \beta_{4}, \gamma_{2}, \gamma_{4}, \beta_{5} \sim N\left(2.95,0.25^{2}\right), \beta_{6} \sim N\left(0.425,0.42^{2}\right), \gamma_{5} \sim$ $N\left(2.15,0.55^{2}\right), \gamma_{6} \sim N\left(0,1.1^{2}\right)$, so that the prior standard deviation was twice of the original prior standard deviation. We assigned $\sigma^{2}$ an $I G(0.01,0.01)$ prior, $\lambda$ and $\kappa \operatorname{Gamma}(0.01,0.01)$ priors, $\gamma_{s 1} \sim U(-3.2,0)$ and $\gamma_{s 2} \sim U(-1.6,1.6)$. The results (see Figure 3 ) are very similar to the original results, suggesting the BILITE is not sensitive to the choice of the prior distributions.

Finally we evaluated the performance of the BILITE design when the true baseline distribution of PFS followed a log-logistic distribution rather than the Weibull distribution. We set the shape parameter at 4 to generate a hump-shaped hazard function. For each scenario, the scale parameter was selected to match the utilities of the two treatments. Results in Figure 3 show that the BILITE design is not sensitive to the distribution of PFS. 


\section{Discussion}

We have developed the BILITE design, a Bayesian phase II randomized design for immunotherapy that jointly models the longitudinal immune response and the time-to-event efficacy. The BILITE design accounts for the important features of immunotherapy that a portion of the patients may develop durable response and that the pattern and magnitude of the longitudinal immune response triggered by immunotherapy treatment is very infor-mative about the progression of the disease. Simulation studies show that the BILITE has desirable operating characteristics and outperforms an alternative design that ignores the immune response. The $\mathrm{R}$ code associated with this paper is freely available by contacting the corresponding author at beibeiguo@lsu.edu.

We focused on two-arm trials, but the BILITE design can be easily extended to multi-arm phase II designs. This is particularly useful due to the recently increasing number of novel

immunotherapeutic agents and their combinations that need to be evaluated in clinical trials. ${ }^{4}$ Also, with simple modification, the BILITE can be used to compare an immunother-apeutic agent with a cytotoxic agent in a two-arm phase II trial setting. The BILITE design can also be extended to incorporate patient biomarkers and other characteristics to achieve "precision medicine". One approach is to include the biomarkers and their interactions with the treatment as covariates into the Cox model and/or the random effects model for the immune response. This is a topic of our future research.

\section{Acknowledgments}

The authors thank two referees for their valuable comments. The research of Beibei Guo is supported by the R \& D Research Competitiveness Subprogram of Louisiana Board of Regents, Contract number LEQSF(2020-21)-RD-A-04. 


\section{References}

[1] Couzin-Frankel J. (2013), Cancer immunotherapy. Science, 324, 1432-1433.

[2] Topalian, S.L., Weiner, G.J. and Pardoll, D.M. (2011), Cancer immunotherapy comes of age. Journal of Clinical Oncology, 23, 4828-4836.

[3] Makkouk, A., and Weiner, G.J. (2015), Cancer immunotherapy and breaking immune tolerance: new approaches to an old challenge. Cancer Research, 75, 5-10.

[4] Yuan, Y., Guo, B., Munsell, M., Lu, K., Jazaeri, A. (2016) MIDAS: a practical Bayesian design for platform trials with molecularly targeted agents. Statistics in Medicine, 35, 3892-906.

[5] Simon R. (1989) Optimal two-stage designs for phase II clinical trials. Controlled Clinical Trials, 10, 1-10.

[6] Chen, T. (1997), Optimal three-stage designs for phase II cancer clinical trials. Statistics in Medicine, 16, 2701-2711.

[7] Ensign LG, Gehan EA, Kamen DS, Thall PF. An optimal three-stage design for phase II clinical trials. Statistics in Medicine 1994; 13:1727-1736.

[8] Hanfelt JJ, Slack RS, Gehan EA. A modification of Simon's optimal design for phase II trials when the criterion is median sample size. Controlled Clinical Trials 1999; 20:555566.

[9] Jung SH, Carey M, Kim KM. Graphical search for two-stage designs for phase II clinical trials. Controlled Clinical Trials 2001; 22:367-372.

[10] Shuster J. Optimal two-stage designs for single arm phase II cancer trials. Journal of Biopharmaceutical Statistics 2002; 12:39-51. 
[11] Lin Y, Shih WJ. Adaptive two-stage designs for single-arm phase IIA cancer clinical trials. Biometrics 2004; 60:482-490.

[12] Thall, P., Simon, R. (1994) Practical Bayesian guidelines for phase IIB clinical trials. Biometrics, 50, 337-349.

[13] Thall PF, Simon RM, Estey EH. Bayesian sequential monitoring designs for single-arm clinical trials with multiple outcomes. Statistics in Medicine 1995; 14:357-379.

[14] Heitjan DF. Bayesian interim analysis of phase II cancer clinical trials. Statistics in Medicine 1997; 16:1791-1802.

[15] Lee JJ, Liu DD. A predictive probability design for phase II cancer clinical trials. Clinical Trials 2008; 5:93-106.

[16] Yin, G., Chen, N., Lee, J. Phase II trial design with Bayesian adaptive randomization and predictive probability. Applied Statistics 2012; 61:219-235.

[17] Johnson VE, Cook JD. Bayesian design of single-arm phase II clinical trials with continuous monitoring. Clinical Trials 2009; 6:217-226.

[18] Guo, B., Zang Y. (2019), A Bayesian adaptive phase II clinical trial design accounting for spatial variation.. Statistical Methods in Medical Research, 28, 3187-3204.

[19] Hodi FS, O'Day SJ, McDermott DF, Weber RW, Sosman JA, Haanen JB, Gonzalez R, Robert C, Schadendorf D, Hassel JC, Akerley W, van den Eertwegh AJ, Lutzky J, Lorigan P, Vaubel JM, Linette GP, Hogg D, Ottensmeier CH, Lebbe C, Peschel C, Quirt I, Clark JI, Wolchok JD, Weber JS, Tian J, Yellin MJ, Nichol GM, Hoos A, Urba WJ. Improved survival with ipilimumab in patients with metastatic melanoma. The New England Journal of Medicine 2010; 363:711-23. 
[20] Robert, C., Schachter, J., Georgina, V.L., and et al. (2015) Pembrolizumab versus Ipilimumab in Advanced Melanoma. The New England Journal of Medicine, 372: 25212532 .

[21] Sato E, Olson SH, Ahn J, Bundy B, Nishikawa H, Qian F, Jungbluth AA, Frosina D, Gnjatic S, Ambrosone C, et al. (2005) Intraepithelial CD8+ tumor-infiltrating lymphocytes and a high $\mathrm{CD} 8+/$ regulatory $\mathrm{T}$ cell ratio are associated with favorable prognosis in ovarian cancer. Proceedings of the National Academy of Sciences of the United States of America, 102, 18538-18543.

[22] Ercolini AM, Ladle BH, Manning EA, Pfannenstiel LW, Armstrong TD, Machiels JP, Bieler JG, Emens LA, Reilly RT, Jaffee EM (2005) Recruitment of latent pools of high-avidity CD8 $+\mathrm{T}$ cells to the antitumor immune response, Journal of Experimental Medicine, 201, 1591-1602.

[23] Galon J, Costes A, Sanchez-Cabo F, Kirilovsky A, Mlecnik B, Lagorce-Pages C, Tosolini M, Camus M, Berger A, Wind P, Zinzindohoue F, Bruneval P, Cugnenc PH, Trajanoski Z, Fridman WH, Pages F. (2006) Type, Density, and Location of Immune Cells Within Human Colorectal Tumors Predict Clinical Outcome Science, 29, 1960-4.

[24] Hamanishi J, Mandai M, Iwasaki M, Okazaki T, Tanaka Y, Yamaguchi K, Higuchi T, Yagi H, Takakura K, Minato N, et al.(2007) Programmed cell death 1 ligand 1 and tumor-infiltrating CD8+ T lymphocytes are prognostic factors of human ovarian cancer, Proceedings of the National Academy of Sciences of the United States of America, 104, $3360-3365$.

[25] Walter S, Weinschenk T, Stenzl A, Zdrojowy R, Pluzanska A, Szczylik C, Staehler M, Brugger W, Dietrich PY, Mendrzyk R, Hilf N, Schoor O, Fritsche J, Mahr A, Maurer D, Vass V, Trautwein C, Lewandrowski P, Flohr C, Pohla H, Stanczak JJ, Bronte V, Mandruzzato S, Biedermann T, Pawelec G, Derhovanessian E, Yamagishi H, Miki T, 
Hongo F, Takaha N, Hirakawa K, Tanaka H, Stevanovic S, Frisch J, Mayer-Mokler A, Kirner A, Rammensee HG, Reinhardt C, Singh-Jasuja H. (2012) Multipeptide immune response to cancer vaccine IMA901 after single-dose cyclophosphamide associates with longer patient survival Nature Medicine, 18, 1254-61.

[26] Zuiverloon TC, Nieuweboer AJ, Vekony H, Kirkels WJ, Bangma CH, Zwarthoff EC. (2012) Markers Predicting Response to Bacillus Calmette-Guerin Immunotherapy in HighRisk Bladder Cancer Patients: A Systematic Review European Urology, 61, 128-145.

[27] Bachmayr-Heyda A, Aust S, Heinze G, Polterauer S, Grimm C, Braicu EI, Sehouli J, Lambrechts S, Vergote I, Mahner S, et al. (2013) Prognostic impact of tumor infiltrating CD8 + T cells in association with cell proliferation in ovarian cancer patients-a study of the OVCAD consortium. BMC Cancer 13:422, doi: 10.1186/1471-2407-13-422.

[28] Law, N., Taylor, J., Sandler, H. (2002) The joint modeling of a longitudinal disease progression marker and the failure time process in the presence of cure. Biostatistics, $\mathbf{3}$, $547-563$.

[29] Brown, E., Ibrahim, J. (2003) A Bayesian semiparametric joint hierarchical model for longitudinal and survival data. Biometrics, 59, 221-8.

[30] Anastasios and Davidian (2004) Joint modeling of longitudinal and time-to-event data: an overview. Statistica Sinica, 14, 809-834.

[31] Yu, M., Taylor, J., Sandler, H. (2008) Individual prediction in prostate cancer studies using a joint longitudinal survival-cure model. Journal of the American Statistical Association 103; 481:178-187.

[32] Song, H., Peng, Y., Tu, D. (2012) A new approach for joint modeling of longitudinal measurements and survival times with a cure fraction. The Canadian Journal of Statistics, 40: $207-224$. 
[33] Li, K., Luo, S. Functional Joint Model for Longitudinal and Time-to-Event Data: An Application to Alzheimer Disease. Statistics in Medicine 2017; 36: 3560-3572.

[34] Bacci, S., Bartolucci, F., Pandolfi, S. (2018) A joint model for longitudinal and survival data based on an $\mathrm{AR}(1)$ latent process. Statistical Methods in Medical Research, DOI: $10.1177 / 0962280216659895$.

[35] Huong, PTT., Nur, D., Pham, H., Branform, (2018) A. A modified two-stage approach for joint modelling of longitudinal and time-to-event data Journal of Statistical Computation and Simulation, 88:3379-3398.

[36] Baart, S., Boersma, E., Rizopoulos, D. (2019) Joint models for longitudinal and timeto-event data in a case-cohort design. Statistics in Medicine, DOI: 10.1002/sim.8113.

[37] Papageorgiou, G., Mauff, K., Tomer, A., Rizopoulos, D. (2019), An Overview of Joint Modeling of Time-to-Event and Longitudinal Outcomes. Annual Review of Statistics and Its Application, 6, 223-240.

[38] Yuan, Y., Yin, G. (2009) JBayesian dose finding by jointly modeling toxicity and efficacy as time-to-event outcomes. Journal of the Royal Statistical Society, Series C, 58, 719-736.

[39] Guo, B., Yuan, Y. (2015), A Bayesian dose-finding design for phase I/II clinical trials with non-ignorable dropouts. Statistics in Medicine, 34, 1721-1732.

[40] Geman, S., Geman, D. (1984) Stochastic Relaxation, Gibbs distributions, and the Bayesian restoration of images. IEEE Transactions on Pattern Analysis and Machine Intelligence, 6, 721-741.

[41] Gelfand, A., Smith, A. (1990) Sampling-Based Approaches to Calculating Marginal Densities. Journal of the American Statistical Association, 85, 398-409. 
[42] Gelman, A., Jakulin, A., Pittau, M. G., Su, Y.S. A weakly informative default prior distribution for logistic and other regression models. The Annals of Applied Statistics 2008; 2: 1360-1383. 
Table 1: Utility for the motivating trial

\begin{tabular}{ccccccc}
\hline & $0 \leq T<3$ & $3 \leq T<6$ & $6 \leq T<9$ & $9 \leq T<12$ & $T \geq 12$ & Cured \\
\hline Utility & 0 & 15 & 30 & 45 & 60 & 100 \\
\hline
\end{tabular}


Table 2: Operating characteristics of BILITE, CPT and CPTO designs. The second column " $\Delta_{\text {true }}$ " in each scenario denotes the "treatment effect", which is the percentage of increase in utility for the experimental treatment relative to the standard treatment. If $\Delta_{\text {true }}>0.3$, the experimental arm is considered superior to the control. The bolded lines represent the true recommended treatment in each scenario.

\begin{tabular}{|c|c|c|c|c|c|c|c|c|c|c|}
\hline & \multirow[b]{2}{*}{$\Delta_{\text {true }}$} & \multicolumn{4}{|c|}{$\mathrm{Z}=0$} & \multicolumn{4}{|c|}{$\mathrm{Z}=1$} & \multirow[b]{2}{*}{$\begin{array}{c}\text { Total } \\
\text { sample } \\
\text { size }\end{array}$} \\
\hline & & Utility & $\begin{array}{c}\text { Selection } \\
\%\end{array}$ & $\begin{array}{c}\text { Number } \\
\text { of } \\
\text { patients }\end{array}$ & $\begin{array}{c}\text { Number } \\
\text { of stage II } \\
\text { patients }\end{array}$ & Utility & $\begin{array}{c}\text { Selection } \\
\%\end{array}$ & $\begin{array}{c}\text { Number } \\
\text { of } \\
\text { patients }\end{array}$ & $\begin{array}{c}\text { Number } \\
\text { of stage II } \\
\text { patients }\end{array}$ & \\
\hline & \multicolumn{10}{|c|}{ Scenario 1} \\
\hline BILITE & 0.52 & 12.7 & 0.341 & 29.9 & 14.9 & 19.3 & 0.659 & 34.7 & 19.7 & 64.6 \\
\hline $\mathrm{CPT}$ & & & 0.385 & 30.1 & 14.9 & & 0.615 & 32.9 & 18.1 & 63.0 \\
\hline \multirow[t]{2}{*}{ СРТO } & & & 0.347 & 28.1 & 13.2 & & 0.653 & 33.2 & 18.2 & 61.3 \\
\hline & \multicolumn{10}{|c|}{ Scenario 2} \\
\hline BILITE & 0.49 & 10.8 & 0.397 & 31.0 & 16.0 & 16.1 & 0.603 & 33.3 & 18.3 & 64.3 \\
\hline $\mathrm{CPT}$ & & & 0.368 & 29.7 & 14.6 & & 0.632 & 32.8 & 17.8 & 62.5 \\
\hline \multirow[t]{2}{*}{ СРTO } & & & 0.422 & 32.2 & 16.9 & & 0.578 & 36.0 & 21.4 & 68.3 \\
\hline & \multicolumn{10}{|c|}{ Scenario 3} \\
\hline BILITE & 0.48 & 8.1 & 0.413 & 30.5 & 15.5 & 12.0 & 0.588 & 32.8 & 17.8 & 63.3 \\
\hline $\mathrm{CPT}$ & & & 0.511 & 33.6 & 18.2 & & 0.489 & 34.6 & 19.9 & 68.1 \\
\hline \multirow[t]{2}{*}{ СРTO } & & & 0.506 & 30.9 & 15.8 & & 0.494 & 32.8 & 17.8 & 63.6 \\
\hline & \multicolumn{10}{|c|}{ Scenario 4} \\
\hline BILITE & 0.68 & 5.6 & 0.359 & 30.3 & 15.2 & 9.4 & 0.641 & 36.1 & 21.1 & 66.3 \\
\hline $\mathrm{CPT}$ & & & 0.410 & 31.7 & 16.6 & & 0.590 & 34.0 & 19.1 & 65.7 \\
\hline \multirow[t]{2}{*}{ СРTO } & & & 0.444 & 31.6 & 16.7 & & 0.556 & 34.6 & 19.4 & 66.1 \\
\hline & \multicolumn{10}{|c|}{ Scenario 5} \\
\hline BILITE & 0.75 & 23.8 & 0.126 & 24.7 & 9.7 & 41.6 & 0.874 & 31.7 & 16.8 & 56.4 \\
\hline CPT & & & 0.235 & 27.2 & 12.2 & & 0.765 & 32.0 & 17.0 & 59.2 \\
\hline \multirow[t]{2}{*}{ СРTO } & & & 0.156 & 23.8 & 8.8 & & 0.844 & 30.2 & 15.3 & 54.0 \\
\hline & \multicolumn{10}{|c|}{ Scenario 6} \\
\hline BILITE & 0.67 & 8.5 & 0.268 & 29.2 & 14.1 & 14.2 & 0.732 & 33.1 & 18.3 & 62.4 \\
\hline $\mathrm{CPT}$ & & & 0.434 & 31.6 & 16.5 & & 0.566 & 33.1 & 18.1 & 64.6 \\
\hline \multirow[t]{2}{*}{ СРТО } & & & 0.472 & 30.6 & 15.6 & & 0.528 & 33.7 & 18.7 & 64.3 \\
\hline & \multicolumn{10}{|c|}{ Scenario 7} \\
\hline BILITE & 0.55 & 9.4 & 0.428 & 33.7 & 18.9 & 14.7 & 0.573 & 36.8 & 21.5 & 70.4 \\
\hline $\mathrm{CPT}$ & & & 0.533 & 33.0 & 18.3 & & 0.467 & 34.2 & 18.9 & 67.2 \\
\hline \multirow[t]{2}{*}{ СРТО } & & & 0.539 & 33.8 & 18.7 & & 0.461 & 33.6 & 18.7 & 67.4 \\
\hline & \multicolumn{10}{|c|}{ Scenario 8} \\
\hline BILITE & 0.11 & 25.7 & 0.806 & 32.8 & 17.8 & 28.5 & 0.194 & 29.0 & 14.0 & 61.8 \\
\hline $\mathrm{CPT}$ & & & 0.734 & 31.6 & 16.5 & & 0.266 & 29.0 & 14.2 & 60.6 \\
\hline \multirow[t]{2}{*}{ СРТО } & & & 0.783 & 33.7 & 18.2 & & 0.217 & 28.8 & 14.3 & 62.5 \\
\hline & \multicolumn{10}{|c|}{ Scenario 9} \\
\hline BILITE & 0.12 & 38.4 & 0.777 & 32.6 & 17.5 & 43.0 & 0.223 & 27.7 & 12.8 & 60.3 \\
\hline $\mathrm{CPT}$ & & & 0.785 & 31.0 & 16.2 & & 0.215 & 26.4 & 11.3 & 57.5 \\
\hline СРТО & & & 0.733 & 31.4 & 16.3 & & 0.267 & 28.9 & 13.9 & 60.3 \\
\hline
\end{tabular}


Table 3: An alternative utility for the sensitivity analysis

\begin{tabular}{ccccccc}
\hline & $0 \leq T<3$ & $3 \leq T<6$ & $6 \leq T<9$ & $9 \leq T<12$ & $T \geq 12$ & Cured \\
\hline Utility & 0 & 25 & 40 & 55 & 70 & 100 \\
\hline
\end{tabular}


Figure 1: Three immune response trajectories generated from model (2) that show increasing, first-increasing-then-plateauing, and first-increasing-then-decreasing trends in the follow-up period $(0,12)$ months. The parameters are $\alpha=20, \eta_{0}=-3, \eta_{1}=0.1, \eta_{2}=0.01 ; \alpha=20, \eta_{0}=$ $-3, \eta_{1}=1, \eta_{2}=0.02$; and $\alpha=20, \eta_{0}=-3, \eta_{1}=1, \eta_{2}=-0.08$ for the three panels from left to right.
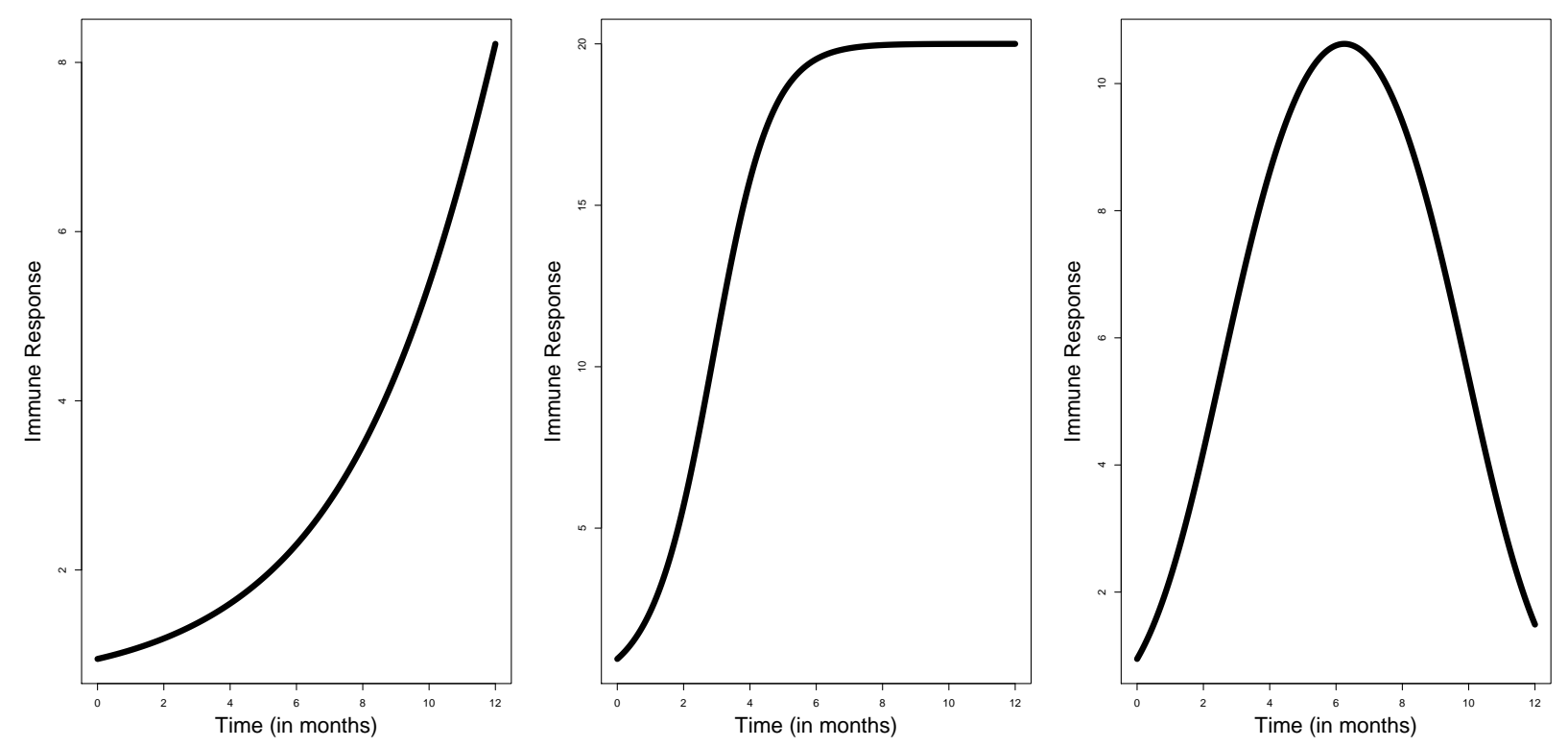
Figure 2: Immune response trajectory for the cured and susceptible subgroups under the two treatments for the 9 scenarios when the random effects $\boldsymbol{b}_{i}$ are 0's. The green and red solid curves represent the cured subgroup under the standard and experimental treatments, respectively; and the green and red broken curves represent the susceptible subgroup under the standard and experimental treatments, respectively.

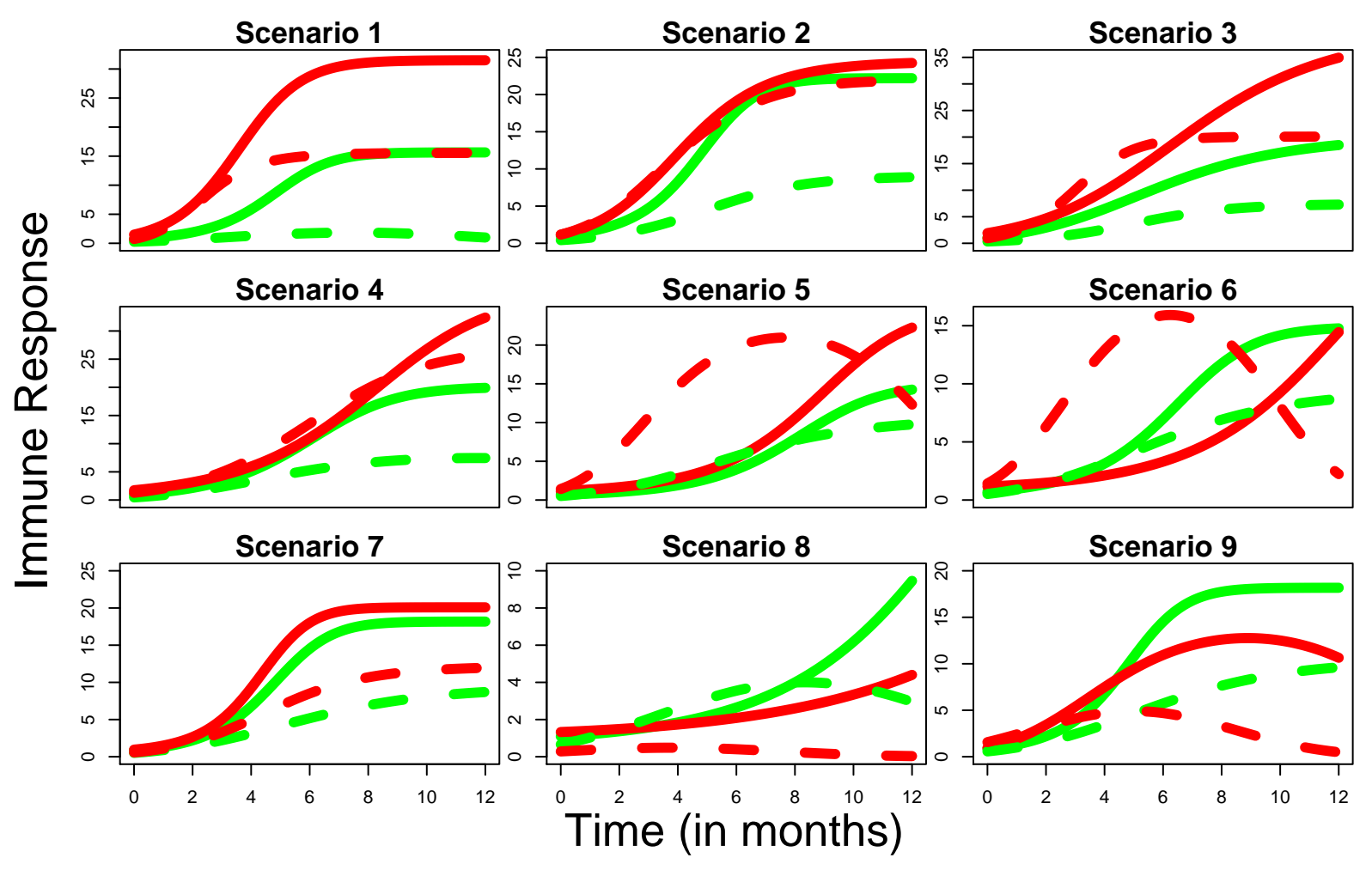


Figure 3: Sensitivity analysis for scenarios 1-6. The top panel is for the selection percentage of the experimental treatment, the bottom panel is for the percentage of patients assigned to the experimental treatment in stage II. In each panel, the $\mathrm{x}$-axis represents the scenario. In each scenario, the five bars represent results with original setting, sample size 60 , an alternative utility, different prior distributions, and log-logistic baseline hazards, respectively, from left to right.

\section{Selection percentage for the experimental treatment}

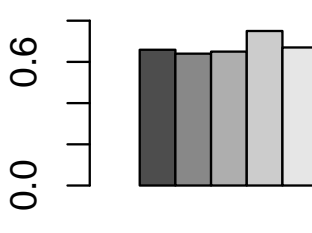

1

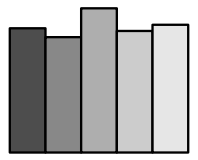

2

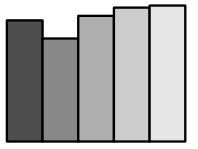

3

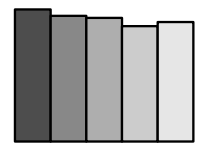

4

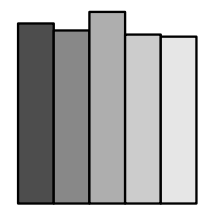

5

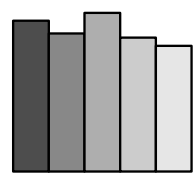

6

Scenario

Percentage of stage II patients assigned to the experimental treatment
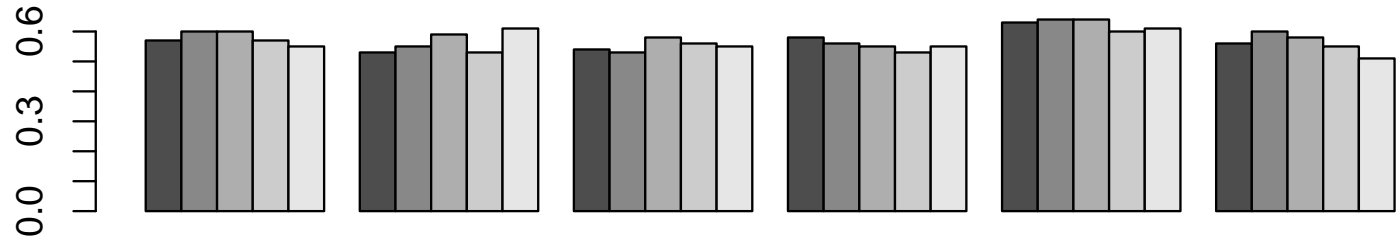

1

2

3

4

5

6

Scenario 